\title{
Preface to the special feature
}

\author{
Akira Namatame ${ }^{1}$
}

Published online: 8 January 2016

(C) Japan Association for Evolutionary Economics 2015

The world economy is changing at an increasing pace in the wake of the ongoing digital revolution. Modern information and communication technologies (ICT) strengthen forces of global flows of goods, people, information, and finance. We live in an increasingly interconnected world in which infrastructures composed of different techno-social system layers. Examples include transportation and mobility infrastructures, global supply chain networks, and financial networks.

Many studies analyze the socioeconomic systems such as cascading failures and financial stability issues using network theory and agent-based modeling. The financial crises, for instance, triggered many studies about systemic risk caused by contagion effects via the interconnections in the global financial networks. Network analysis provides powerful mathematical tools for analyzing the global circulation of goods, resources, people, money, and information. The accessibility of new data and the advances in theory and modeling are providing an integrated framework that brings us closer to analyzing the behavior of socioeconomic systems from a new perspective. An agent-based approach has also proven useful in many applications. Agent-based modeling provides answers to questions where traditional analysis methods are weak, and it is leading to improved models across wide types of financial risks. This special issue is being launched at a time of ever-increasing connectivity and complexity in socioeconomic networks.

The first article in the issue, "Towards capturing heterogeneity of supply network structures and their temporal transitions: an investigation of supply relationships in the Japanese automobile industry" by Tomomi Kito and Steve New, consider connectivity among car manufacturers (OEMs) and auto-parts suppliers in the

Akira Namatame

nama@nda.ac.jp

1 Department of Computer Science, National Defense Academy, Yokosuka, Japan 
Japanese automobile industry. They have investigated, using large-scale longitudinal data, the heterogeneous formations and the temporal evolutions of the supply networks of various products. To elucidate structural variations in the supply networks, they identify the following as measures for characterization of each product market: the number of suppliers, the number of supply relationships, and the variance in the numbers of supply relationships that suppliers have. In the past, the average numbers of OEM clients per supplier have been surveyed; however, they point out that this number varies significantly from supplier to supplier, and the variance of this number is essential to capture the characteristics of the product market. By capturing product markets via the new measures and observing the transitions over time, they obtained general insight into the conditions of matured and stable markets as well as competitive and unstable ones. They also identified how the market selection process may affect the transition of the market characteristics, which enable us to predict how the markets will change over time. Most importantly, their article shows how methodologies from network theory can help reformulate long-standing questions in global supply chains and provide new insights.

The issue's second article, "Worldwide aviation network vulnerability analysis: a complex network approach" by Hoang Anh Quang Tran and Akira Namatame, investigates the worldwide aviation networks (WANs). The flight data for 2014 was obtained from the open source project named OpenFlights. They clarify that WANs have heterogeneous distributions of degree, strength, and betweenness, which follow a double power law. The WANs are also a small world with about 28 community structures. Their analysis provides a closer look at how the worldwide air transportation system has been constructed and how it responds to catastrophic events. They investigate the cascade dynamics of the network, with emphasis on the understanding of the resilience of the WANs to extreme events such as intentional attacks or disruptions with severe weather conditions. Topological integrity, which is widely used to assess the vulnerability of complex networks, is not suitable for the study of robustness of aviation networks, and they introduce a new measurement. They show that centrality-driven attacks lead to a massive loss of aviation network efficiency, even at very low levels of damage in the connectivity pattern. That is to say, central airports based on the new measurement have a strong impact on network resilience, and the failure of one of them results in serious consequences to the operation of the entire aviation network. This provides a clear identification of the aviation network needs in the new mitigation strategies against such intentional attacks or disruptions.

The third article, "Effects of dark pools on financial markets' efficiency and price discovery function: an investigation by multi-agent simulations" by Takanobu Mizuta et al., provides very rich insight into the complex multiplex nature of financial networks and helps to understand and model how these networks interact in countries where data at such a detailed level are not available, or where the data are confidential. The authors investigate how a dark pool, in which no order books are provided, affects financial markets' efficiency and price-discovery function by using the agent-based model. This is an important investigation into financial systemic risk because making a market inefficient and losing the price-discovery function may make the market unstable and increase financial systemic risk. They 
also discuss quantitatively how the spreading of dark pools beyond our experience can affect the price-discovery function. They aim to clarify the mechanism of a dark pool that makes a market efficient or inefficient. The agent-based model should replicate these values as significantly positive and within a reasonable range.

The issue's fourth article, "Shock transfer by arbitrage trading: analysis using multi-asset artificial market" by Takuma Torii et al., addresses the problem of new trading technologies, such as high-frequency and algorithmic trading, which were invented to promote simultaneous trading of multiple assets. Simultaneous trading is often regarded as a major cause of mimicking the price movements of each other just before a financial crisis. For instance, a number of stocks trading multiple assets is becoming more common in financial markets, but financial analysts argue that it may bring unintended consequences, such as an increase in volatility. The authors show that agent-based modeling is useful in order to study market dynamics and acquire information to devise market rules.

All of these contributions cast new light on various aspects of integrating network science and agent-based modeling. These articles have quite early origins and they shed light on newly emerging economic questions. I hope this special issue will foster links among the community of researchers interested in socioeconomic networks and agent-based modeling, and that the techniques and findings presented herein find valuable application among practitioners. 